\title{
An Entropy Measurement Method of Quantum Information System under Uncertain Environment
}

\author{
Zijing Li' ${ }^{1}$, Jiangnan Deng², Bing Zhou ${ }^{3}$, Ying Liu ${ }^{4}$, Zhengying Cai1 ${ }^{*}$ \\ ${ }^{1}$ College of Computer and Information Technology, China Three Gorges University, Yichang, China \\ ${ }^{2}$ College of Law and Public Administration, China Three Gorges University, Yichang, China \\ ${ }^{3}$ College of Biological and Pharmaceutical Sciences, China Three Gorges University, Yichang, China \\ ${ }^{4}$ School of Foreign Language, China Three Gorges University, Yichang, China \\ Email: 1483613822@qq.com,1394720486@qq.com,1050015785@qq.com,1311264797@qq.com,*master_cai@163.com
}

How to cite this paper: Li, Z.J., Deng, J.N., Zhou, B., Liu, Y. and Cai, Z.Y. (2018) An Entropy Measurement Method of Quantum Information System under Uncertain Environment. International Journal of Intelligence Science, 8, 29-41.

https://doi.org/10.4236/ijis.2018.82002

Received: February 1, 2018

Accepted: April 27, 2018

Published: April 30, 2018

Copyright $\odot 2018$ by authors and Scientific Research Publishing Inc. This work is licensed under the Creative Commons Attribution International License (CC BY 4.0).

http://creativecommons.org/licenses/by/4.0/

\begin{abstract}
Under uncertain environment, it is very difficult to measure the entropy of quantum information system, because there is no effective method to model the randomness. First, different from the traditional classic uncertainty, a quantum uncertain model is proposed to simulate a quantum information system under uncertain environment, and to simplify the entropy measurement of quantum information system. Second, different from the classic random seed under uncertain environment which is often called as pseudo-random number, here the quantum random is employed to provide us a true random model for the entropy of quantum information system. Third, the complex interaction and entangling activity of uncertain factors of quantum information is modeled as quantum binary instead of classic binary, which can help us to evaluate the entropy of uncertain environment, to analyze the entropy divergence in quantum information system. This work presents a non-classic risk factor measurement for quantum information system and a helpful entropy measurement.
\end{abstract}

\section{Keywords}

Entropy Measurement Method, Quantum Information System, Uncertain Environment, Quantum Encryption

\section{Introduction}

\subsection{Related Work}

At present, the information system under uncertain environment is a hot point 
in research. [1] used a multi-AUV autonomous task planning based on the scroll time domain quantum bee colony optimization algorithm in uncertain environment, and [2] unveiled the performance verification for robot missions in uncertain environments. Different researchers provided different models. [3] sketched the quantum information splitting of an arbitrary three-ion state in ion-trap system, and [4] sculpted a dynamics of quantum fisher information in a two-level system coupled to multiple bosonic reservoirs. After that [5] reviewed the long-distance quantum information transfer with strong coupling hybrid solid system.

The appearance of quantum mechanics brought more uncertainty in information system which is different from traditional classic uncertainty. [6] researched Classical Information Storage in an n-Level Quantum System, and [7] implied a certifying single-system steering for quantum-information processing. Then, [8] illustrated causal boxes: quantum information-processing systems closed under composition, and [9] gave the dynamics of entanglement and quantum Fisher information for $\mathrm{N}$-level atomic system under intrinsic decoherence. Apparently, the entanglement activity brought more uncertainty into quantum information. [10] framed information propagation in isolated quantum systems, and [11] featured noise management to achieve superiority in quantum information systems. [12] exhibited dynamics of quantum information in many-body localized systems, and [13] evaluated holographic control of information and dynamical topology change for composite open quantum systems, [14] extended thermodynamic description of non-Markovian information flux of nonequilibrium open quantum systems.

The entropy measurement method provided us a useful tool to measure the uncertain information. [15] etched a novel measurement method for transient detection based in wavelets entropy and the spectral kurtosis and provided an application to vibrations and acoustic emission signals from termite activity. [16] drew a method for the estimation of standard entropy of crystal phases at 298.15 $\mathrm{K}$ on the limited temperature range of heat capacity measurements, and [17] constructed a measurement method of differences in group preferences based on relative entropy. [18] developed virtual entropy generation (VEG) method in experiment reliability control with implications for heat exchanger measurement, and [19] discussed a bi-objective MIP model for facility layout problem in uncertain environment. These researches gave us a useful tool to evaluate the entropy of uncertain systems with classic mathematics.

After that the quantum entropy was put forward. [20] depicted a mean-reverting currency model in an uncertain environment, and [21] proposed an efficient systolic array grid-based structure of the robust Bayesian regularization technique for realtime enhanced imaging in uncertain remote sensing environment. [22] presented a dynamical scheduling and robust control in uncertain environments with petri nets for DESs. [23] proposed a discontinuity and protection of quantum fisher information for a two-qubit system. These researches illuminated that the entropy can be employed to measure the uncertainty of informa- 
tion system. However, these previous researches above focused on classic entropy measurement of information system, this paper tried to provide a method to measure the entropy of quantum information system by quantum entropy.

\subsection{Organization of the Article}

In Section 2, modelling a quantum information system is defined. Preliminaries, In Section 3, entropy of quantum information system will be contained. In Section 4, the entropy measurement of quantum information system will be described. In Section 5, the improvement for quantum information system will be contained.

\section{Modelling a Quantum Information System}

\subsection{Operation of Quantum Information Systems}

In quantum information system, there are full of quantum binary data as information flow. Once a information sequence defines, a series of quantum binary data will be used to refer specially appointed information (e.g., $d_{1}, \cdots, d_{y}$ ) and the context will be used to refer to the information sequence as a whole (e.g., $d$ ). For each $b$, let $b=1-b$. For each sequence of bits $b=\left(b_{1}, \cdots, b_{y}\right)$, let $\bar{b}=\left(\bar{b}_{1}, \cdots, \bar{b}_{y}\right)$.

The form $f(m) n$ (where $f$ is a function) is supposed to mean $(f(m)) n$. Therefore, such as, in the form

$$
\gamma r[\mathrm{Z}] 1 / q
$$

Rather before it, after the trace function the $(1 / q)$ th power map is displays.

$(\log m)$ is used to express the logarithm with base 2, and $(\ln m)$ is used to express the logarithm with base e.d: $[0,1] \rightarrow \mathrm{R}$ is used to express the Shannon entropy function of quantum information system [3]:

$$
d(m)=-m \log m-(1-m) \log (1-m)
$$

Capital letters (e.g., $Q$ ) are used to express quantum information systems. The same letter are used to express not only the system itself but also the complicated Hilbert space to be indicated. Each limited dimensional complicated Hilbert space $Q, L(Q)$ is expressed to be the set of linear maps very $Q$ to itself, and let

$$
\begin{gathered}
P(Q)=\{A \in \rho(Q) \mid A \geq 0\} \\
S(Q)=\{A \in \rho(Q) \mid A \geq 0, \gamma r(A) \leq 1\} \\
H(Q)=\{A \in \rho(Q) \mid A \geq 0, \gamma r(A)=1\}
\end{gathered}
$$

All these above are, respectively, a group of positive semidefinite operators, a group of subnormal-sized positive semidefinite operators, and a group of density operators.

Suppose that $L_{1}: M_{1} \rightarrow N_{1}$ and $L_{2}: M_{2} \rightarrow N_{2}$ are two linear operators, so that the 
operator from $M_{1} \oplus M_{2}$ to $N_{1} \oplus N_{2}$ that maps $\left(m_{1}, m_{2}\right)$ to $\left(L_{1}\left(m_{1}\right), L_{2}\left(m_{2}\right)\right)$ are devoted by $L_{1} \oplus L_{2}$.

Suppose that $(\alpha, E)$ is a bipartite system, and $L$ is supposed to be a density operator about $\alpha \otimes E$ to show a traditional-quantum state, thus $L$ can be used as a diagonal-block operator [12]

$$
L=\left|\begin{array}{ccccc}
L_{1} & & & & \\
& L_{2} & & & \\
& & L_{3} & & \\
& & & \ddots & \\
& & & & L_{x}
\end{array}\right|
$$

In quantum information system, the subnormalized operators of $E$ reply to the basis groups of the classical register $B$ are denoted by $L_{1}, \cdots, L_{x}$. Additionally, $L$ can be expressed as $L=L_{1} \oplus L_{2} \oplus \cdots \oplus L_{x}$.

Whichever $B>0$, as well as each linear operator $M$, the Schatten norm is denoted by $\|M\|_{B}$ :

$$
\|M\|_{B}=\gamma r\left[(M * M)^{B / 2}\right]^{1 / B} .
$$

Moreover, for $M$ is positive semidefinite, that this may be written more easily as

$$
\|M\|_{B}=\gamma r\left[M^{B}\right]^{1 / B}
$$

Now the quantum information system can be expressed as Equation (8). When $m \in[0,2]$, the function $Z \mapsto Z m$ matters frequently. Let $T \in[0,1]$, and let $Z, W$ are denoted positive semidefinite operators about $\mathrm{C}^{y}$.

(a) If $Z \leq W$, then $Z T \leq W T$.

(b) If $Z \leq W$ and $X=W-Z$, then

$$
\gamma\left(M_{1}+T\right)+\gamma r\left(Z_{1}+T\right) \leq \gamma r\left(W_{1}+T\right)
$$

Part (a) are followed by part (b) in quantum information system by the following reasoning:

$$
\begin{aligned}
& \gamma r\left(W_{1}+T\right)=\gamma r(W \cdot W T) \\
= & \gamma r(M \cdot W T)+\gamma r(Z \cdot W T) \\
\geq & \gamma r(M \cdot X T)+\gamma r(Z \cdot Z T) \\
= & \gamma r\left(M_{1}+T\right)+\gamma r\left(Z_{1}+T\right)
\end{aligned}
$$

\subsection{The Interaction of Quantum Binary and Classic Binary}

As a finite dimensional C-vector space in quantum information system, and $M_{0}$, $M_{1}$ is supposed to be Hermitian operators about binary vector $V$ satisfying $\left\|M_{0}\right\|,\left\|M_{1}\right\| \leq 1$. Thus, there exists a unitary embedding $U: W \rightarrow C^{2 y}, y \geq 1$, and operators $N_{0}, N_{1}$ of the form [3] 


$$
N_{0}=\left[\begin{array}{ccccccc}
0 & 1 & & & & & \\
1 & 0 & & & & & \\
& & 0 & 1 & & & \\
& 1 & 0 & & & \\
& & & \ldots & & \\
& & & & 0 & 1 \\
& & & & 1 & 0
\end{array}\right], N_{1}=\left[\begin{array}{ccccccc}
0 & \zeta_{1} & & & & & \\
\frac{\zeta_{1}}{1} & 0 & & & & & \\
& & 0 & \zeta_{2} & & & \\
& \frac{\zeta_{2}}{3} & 0 & & & \\
& & & \ddots & & \\
& & & & 0 & \zeta_{x_{j}} \\
& & & & \frac{\zeta_{x_{j}}}{3} & 0
\end{array}\right]
$$

with $\left\|\varsigma_{k}\right\|=1$, so that $M_{k}=U^{*} N_{k} U$ for $k \in\{0,1\}$.

Considering the condition of quantum information system, binary information flow is satisfied by the class of all triples $\left(V, M_{0}, M_{1}\right)$. On the following triples consider the following two conditions:

a) $M_{k} M_{k}^{2}=I$ is satisfied by the operators

b) Being equal to $\mathrm{C}^{x}$, the vector space $V$ and $M_{0}, M_{1}$ are considered as a block form that is uniform diagonal:

$$
M_{K}=\left[\begin{array}{cccccccc}
\alpha_{k}^{1} & & & & & & & \\
& \alpha_{k}^{2} & & & & & & \\
& & \ddots & & & & & \\
& & & \alpha_{k}^{r} & & & & \\
& & & & b_{k}^{1} & & & \\
& & & & & b_{k}^{2} & & \\
& & & & & & \ddots & \\
& & & & & & & b_{k}^{s}
\end{array}\right]
$$

Consider that $b_{k}^{j} \in\{-1,+1\}$ and each $\alpha_{k}^{j}$ is a $2 \times 2$ Hermitian matrix with eigenvalues +1 and -1 at the condition $2 r+s=x$,

For each triple $\left(V, M_{0}, M_{1}\right)$ has a unitary embedding to a triple that satisfies the conditions of quantum binary system, as well as Condition $(\sigma)$.

Let $U(v)=v \oplus 0$ gives $U: V \rightarrow V \oplus V$, and $\left\{M_{k}^{\prime} \mid k=0,1\right\}$ is considered to be the operators about $V \oplus V$ defined by [12]

$$
M_{k}^{\prime}=\left[\begin{array}{cc}
M_{k} & \sqrt{1-M_{k}^{2}} \\
\sqrt{1-M_{k}^{2}} & -M_{k}
\end{array}\right]
$$

That $\left(M_{k}^{\prime}\right)^{2}=$ II is easily to be checked.

For each triple $\left(V, M_{0}, M_{1}\right)$ has a unitary embedding into a triple that satisfying Condition $(\sigma)$ as well as Condition $(\alpha)$.

An orthonormal basis $\left\{v_{1}, \cdots, v_{\operatorname{dim} V}\right\}$ can be chosen for $V$ so that $M_{0}$ has the form $M_{0}=\left[\begin{array}{cc}\Pi_{n} & 0 \\ 0 & -\Pi_{m}\end{array}\right]$ where the $r \times r$ binary identity matrix is denoted by $I_{r}$ An opportune unitary transformation of $V$ that represents this information system structure contains another orthonormal basis $\left\{v_{1}^{\prime}, \cdots, v_{\operatorname{dim} v}\right\}$ thus $M_{0}$ and $M_{1}$ have the form

$$
M_{0}=\left[\begin{array}{cc}
\Pi_{n} & 0 \\
0 & -\Pi_{m}
\end{array}\right] \text { and } M_{1}=\left[\begin{array}{cc}
A & D \\
D^{*} & C
\end{array}\right]
$$


Let $\alpha$ and $C$ to be diagonal matrices. That $\sigma^{2}+H H^{*}=I I$ and $H^{*} H+C^{2}=I I$ are implied by the condition $M_{1}^{2}=$ II . For both $H H^{*}$ and $H^{*} H$ are diagonal, as well as $H$. For each triple $\left(V, M_{0}, M_{1}\right.$ ) has a unitary embedding into a triple of the form satisfying Condition (a).

It can be proved enough that $M_{0}, M_{1}$ are both scalars different from classical binary information system, and $M_{0}, M_{1}$ are each $2 \times 2$ Hermitian matrices with eigenvalues +1 and -1 . For the quantum information system, an orthonormal basis $\left\{v_{1}, v_{2}\right\}$ for $C^{2}$ under which $M_{0}=\left[\begin{array}{ll}0 & 1 \\ 1 & 0\end{array}\right]$ can be found, then under $M_{1}$ there is an antidiagonal matrix, a set of the form

$\left\{(\cos \theta) v_{1}+i(\sin \theta) v_{2},(\cos \theta) v_{2}+i(\sin \theta) v_{1}\right\} \quad$ with $\quad z \in \mathrm{C}, \quad|z|=1 \quad$ can be found.

\section{Entropy of Quantum Information System}

It is a challenge to evaluate the uncertainty in quantum information system. Although entropy can be used to evaluate the uncertainty of information system with a classical number, such as the possibility $B$. Often a possibility is between o and 1 , but add or subtract each other by information interaction. For $B$ is too close to 1 , then in the inequality associated with $D B$ to $D_{\min }^{\lambda}$ the penalty term will be big enough so that the lower bound of the smooth minimum entropy will be invalid, but this kind of operation may be not effective to evaluate the entropy of quantum information system. For $B$ is too far from 1, such as Game Round Agreements R, the Renyientropy will not be enough sensitive so that detecting the effect of small probability events becomes harder to give us the entropy of quantum information system. Roughly speaking, the parameter $B$ which is set such that the parameter $q$ is proportional to $B-1$ which is thus appropriate according for parameters in the entropy measurement.

To solve the entropy measurement problem, a quantum entropy method and measurement are built based on an entangled qubit. If $Q E$ is a two-part system at $Q=2$, then it is considered that $\{L 0, L 1\}$ and $\{L+, L-\}$ are respectively the calculation base of $Q$ and the subnormalizing state of Hadamard base $E$. On the quantities $\gamma r\left[L_{M}^{1+\lambda}\right]$, uniform constraints (independent of the dimension of $E$ ) are expressed by Theorem 4.2. So that other known uncertainty relations such as quantum entanglement are paralleled.

To prove the effectiveness of $\sigma^{\prime}$, it is considered the quantum entropy as $\sigma^{\prime}$ that $G=\left(G_{1}, \cdots, G_{Y}\right)$ and $O=\left(O_{1}, \cdots, O_{Y}\right)$ which are registers respectively contains the input bits and output bits, where $\sigma^{\prime}, E$ is considered to express a purifying system for the device. According to the "success" event $(s), \Gamma_{E G O}^{s}$ is considered to express the subnormalized state of these three systems, with an upper bound on the (negative) quantity $H_{B}\left(\Gamma_{E G O}^{s} \| \Gamma_{E G} \otimes \mathrm{II}_{O}\right)$.

The first-round registers $G_{1}$ and $O_{1}$ are considered. On $E G_{1} O_{1}$ the bounding operator $\Gamma_{E G_{1}} \otimes \mathrm{II}_{O_{1}}$ is equal to

$$
\begin{aligned}
& (1-q) \Gamma_{E} \otimes|00\rangle\left\langle 00\left|+(1-q) \Gamma_{E} \otimes\right| 01\right\rangle\langle 01| \\
& +(q) \Gamma_{E} \otimes|10\rangle\left\langle 10\left|+(q) \Gamma_{E} \otimes\right| 11\right\rangle\langle 11| .
\end{aligned}
$$


$\varepsilon>0$ is considered as a real parameter, and in the entropy summand, the following is considered to be alternative operator, which the factor $2 \varepsilon$ is inserted in to be:

$$
\begin{aligned}
\Sigma:= & (1-q) \Gamma_{E} \otimes|00\rangle\left\langle 00\left|+(1-q) \Gamma_{E} \otimes\right| 01\right\rangle\langle 01| \\
& +(q) \Gamma_{E} \otimes|10\rangle\left\langle 10\left|+(q) 2^{\varepsilon} \Gamma_{E} \otimes\right| 11\right\rangle\langle 11| .
\end{aligned}
$$

When the entropy $(g, o)=(1,1)$ occurs corresponding to a game-loss in quantum information system $R$, randomness is added by the factor $2 \varepsilon$ artificially. According to how well the device performs, the expectation for randomness is lowered effectively.

For appropriate $\mathcal{E}, B$, the amount $H B\left(r E G_{1} O_{1} \| \Sigma\right)$ has a form upper bound less than zero implied by the uncertainty principle for quantum entropy. By the earlier reduction discussed, a lower bound on the amount of extractable bits output by quantum information entropy $R$ used.

\section{Entropy Measurement of Quantum Information System}

Let $S$ expresses uncertain entanglement in entropy measurement where information system exists, and let $\Gamma_{E G O}^{s}$ expresses the corresponding (subnormalized) operator on $E \otimes G \otimes O$.

$A$ continuous entangled entropy function $R(v, d, \mu, q, \kappa, r)$ exists as shown below.

1) Let $\lambda>0$. If a quantum information system $\sigma^{\prime}$ is performed with parameters $(v, d, Y, q, \mu, H)$, then

$$
D_{\min }^{\lambda}\left(\Gamma_{E G O}^{s} \mid E G\right) \geq Y \cdot R(v, d, q, \kappa, r)-\frac{\log \left(2 / \lambda^{2}\right)}{q \kappa r}
$$

2) The following equality exists:

The uncertainty of system $\sigma$ is defined by an inequality

$$
\lim _{(q, \kappa) \rightarrow(0,0)} R(v, d, \mu, q, \kappa, r)=\min _{s \in[0,1]}\left(\pi(s)+\frac{v s-\mu}{r}\right) .
$$

The success of a quantum information system $\sigma$ is defined by an inequality

$$
\sum_{i} g_{i} o_{i} \leq(d / 2+\mu) q Y
$$

Make $S \subseteq G \otimes O$ is the scope of the vector $|g o\rangle$ of $(G, O)$ in Equation (20) between all of the sequence of change.

And for any classical quantum operator, $X$ on $E \otimes G \otimes O$ with respect to $(G O \mid E)$, let $M^{s}$ represent the restriction of $M$ to $E \otimes S M$ to $E$. Applying this structure to inferences Corollary 7.5, to the operators $\Gamma_{E G O}, \bar{\Gamma}_{E G O}$ and using the fact that the constraints of $H_{\max }$ and $\|\cdot\|_{1}$ and $\mathrm{S}$ are monotonically reduced, it can be found that [12].

$$
H_{\max }^{\lambda}\left(\Gamma_{E G O}^{s} \| \Sigma^{s}\right) \leq-Y \cdot R(v, d, q, \kappa, r)+\frac{\log \left(2 / \lambda^{2}\right)}{q \kappa r}
$$

To smooth the lower bound of minimum entropy of $\Gamma_{E G O}^{s}$ is given, where $X$ 
is a density matrix, it is needed with respect to calculate to an operator on $E \otimes G \otimes O$ that is of the form $M \otimes \mathrm{II}_{o}$ on the divergent. Suppose a new operator $N /$ on $E \otimes G \otimes O$ by

$$
\Sigma^{\prime}=\Gamma_{E} \otimes\left(\sum_{(g, o) \in S}(1-q)^{\sum_{i}\left(1-g_{i}\right)} q^{\sum_{i} g_{i}} 2^{(d / 2+\mu) / Y r}|g o\rangle\langle g o|\right)
$$

(recalling that $T=q \kappa r$ ). Comparing this definition with Equation (22) and applying the success standard Equation (21), it can be found that $\Sigma^{\prime} \geq \Sigma^{s}$. Thus, the boundary in Equation (22) is also established, when $\Sigma^{s}$ is replaced by $\Sigma^{\prime}$.

When suppose that $\Psi$ as the operator on $E \otimes G$ defined by

$$
\Psi=\Gamma_{E} \otimes \sum_{g, o \in\{0,1\}}(1-q)^{\sum_{i}\left(1-g_{i}\right)} q^{\sum_{i} g_{i}}|g\rangle\langle g|
$$

and rewrite $\Sigma^{\prime}$ as

$$
\Sigma^{\prime}=2^{(d / 2+\mu) / Y / r}\left(\Psi \otimes \mathrm{II}_{o}\right)
$$

It can be found (applying the rule $\left.H_{\max }^{\lambda}(X \| Y)=\log c+H_{\max }^{\lambda}(X \| c Y)\right)$ that

$$
H_{\max }^{\lambda}\left(\Gamma_{E G O}^{s} \| \Psi \otimes \mathrm{II}_{O}\right) \leq(d / 2+\mu) Y / r-Y \cdot \Delta(v, d, q, \kappa, r)+\frac{\log \left(2 / \lambda^{2}\right)}{q \kappa r} \text { that }
$$

For $\Psi$ is a density matrix, then that

$$
D_{\text {min }}^{\lambda}\left(\Gamma_{E G O}^{s} \mid E G\right) \geq-H_{\max }^{\lambda}\left(\Gamma_{E G O}^{s} \| \Psi \otimes \mathrm{II}_{O}\right)
$$

Therefore, if it is supposed that

$$
R(v, d, q, \kappa, r)=-\frac{d / 2+\mu}{r}+\Delta(v, d, q, \kappa, r)
$$

Condition 1 of the theorem is satisfied. Condition 2 is easily obtained from the limit formula of limit of $\sigma(22)$.

By optimizing the coefficient $r$, it can be made a final improvement on the previous results $\sigma$.

There exist continuous real-valued functions $\gamma(v, d, \mu, q, \kappa)$ and $F(v, d, \mu, q, \kappa)$ so that the following holds.

1) If Protocol $\sigma^{\prime}$ is performed with parameters $(v, d, Y, q, \mu, \kappa)$, thus for any $\lambda \in(0, \sqrt{2}]$ and $\kappa \in(0, \infty)$,

$$
D_{\min }^{\lambda}\left(\Gamma_{E G O}^{s} \mid E G\right) \geq Y \cdot T(v, d, \mu, q, \kappa)-\left(\frac{\log (\sqrt{2} / \lambda)}{q \kappa}\right) F(v, d, \mu, q, \kappa)
$$

2) The following equalities hold, where $n$ expresses the function from Theorem 4.2 .

$$
\begin{gathered}
\lim _{(q, \kappa) \rightarrow(0,0)} T(v, d, \mu, q, \kappa)=\pi(\mu / v) \\
\lim _{(q, \kappa) \rightarrow(0,0)} F(v, d, \mu, q, \kappa)=\frac{-2 \pi^{\prime}(\mu / v)}{v}
\end{gathered}
$$

Let 


$$
r=\min \left\{\frac{v}{-\pi^{\prime}(\mu / v)}, \frac{1}{q \kappa}\right\}
$$

Define the function $T$ by [11]

$$
T(\nu, d, \mu, q, \kappa)=R(\nu, d, \mu, q, \kappa) .
$$

By substitution into quantum information system (28), the constraint Equation (31) will be established when $F$ is set to equal to 2 (r). To measure the entropy of quantum information system, please note.

$$
\begin{aligned}
\lim _{(q, \kappa) \rightarrow(0,0)} T(v, d, \mu, q, \kappa) & =\lim _{(q, \kappa) \rightarrow(0,0)} R\left(v, d, \mu, q, \kappa, \frac{v}{-\pi^{\prime}(\mu / v)}\right) \\
& =\min _{s \in[0,1]}\left[\pi(s)-\frac{-\pi^{\prime}(\mu / v)}{v}(v s-\mu)\right] \\
& =\min _{s \in[0,1]}\left[\pi(s)-\frac{-\pi^{\prime}(\mu / v)}{v}\left(s-\frac{\mu}{v}\right)\right]
\end{aligned}
$$

By square brackets in Equation (32), the function that is enclosed is a convex function of $s$ whose derivative at $s=\mu / v$ is zero. Thus, at $s=\mu / v$, a minimum is achieved and the expression in Equation (32) therefore expression in the assessment simply to $\pi(\mu / v)$.

\section{Improvement for Quantum Information System}

To improve the stability of quantum information system, the system evolution should march toward the direction of the minimization of entropy. Suppose that $U \subseteq R^{y}$, Let $z \subseteq R^{y}$ as an element in the closure of $U$, and suppose $f, g$ as continuous functions from $U$ to $R$. Let that

$$
\lim _{M \rightarrow z} f(M)=0
$$

And

$$
\lim _{M \rightarrow z} \frac{f(M)}{g(M)}=c
$$

Then,

$$
\lim _{M \rightarrow Z}(1+f(M))^{1 / g(M)}=\mathrm{e}^{c}
$$

It is easily to prove by taking the natural logarithm of two sides of Equation (34), which can direct the evolution of system entropy.

Suppose that $U \subseteq R^{y}$ and $Y \subseteq R^{x}$, and denote that $V$ is compact. Suppose $f: U \times V \rightarrow R$ as a continuous function. Suppose in the closure of $U$ z $\in R^{y}$ as an element, and denote that for every $n_{0} \in V \quad \lim _{(m, n) \rightarrow\left(z, y_{0}\right)} f(m, n)$ exists. Thus,

$$
\lim _{M \rightarrow Z} \min _{n \in V} f(M, N)=\min _{n \in V} \lim _{m \rightarrow Z} f(M, N)
$$

By assumption, a continuous extension of $f$ to $(U \bigcup\{z\}) \times V$ exists. By $\bar{f}$ this extension can be expressed. Suppose that $d(m, n)=\bar{f}(m, n)-\bar{f}(z, n)$.

Let $\delta>0$. Since $h(z, n)=0$ and $d$ is continuous at $(z, n)$ for any $n \in V$, it can 
be found that an $\lambda_{n}>0$ so that the values of $d$ on the cylinder

$$
\left\{\left(m, n^{\prime}\right)|| m-z\left|<\lambda_{n},\right| n^{\prime}-n \mid<\lambda_{n}\right\}
$$

are defined to $[-\delta, \delta]$. Because $V$ is compact, it can be chosen that a finite set $S \subseteq$ $V$ making the positive $\lambda_{n}-c_{n}$ linders for $n \in S$ cover $V$. Supposing $\lambda=\min _{n} \lambda S \lambda_{n}$, it can be found that the values of $d$ on the $\lambda$-neighborhood of $V$ are defined to $[-\delta, \delta]$. Thus, the minimum value of $f(m, n)$ on the $\in-$ neighborhood of $V$ is in the range $\delta$ of $\min _{n \in v} \bar{f}(z, n)$.

Supposing $H$ is a binary quantum device with a y component in entropy evolution process. For any $j \geq 1$, it can be used that the expression $I_{j}$ and $N_{j}$ to represent the input strings and output strings of $H$ in the $t$ th iteration (each string is in $\left.\{0,1\}^{x}\right)$.

Suppose that $G$ as a powerful self-test. For each input quantum information $i=\left(i^{1}, \cdots, i^{y}\right) \in\{0,1\}^{y}$, the unique optimal strategy of the output information is determined by the unique optimal strategy to minimize the entropy with the output information $n \in\{0,1\}^{y}$ that it can be denoted by $P_{i}^{n} \mid n \in\{0,1\}^{y}$. It can be said that if, for any $k \geq 1$, and $i_{1}, \cdots, i_{k}, n_{1}, \cdots, n_{k-1} \in\{0,1\}^{y} \quad H$ has noise level $\beta$ (for the game $G$ ) so that [11]

$$
P\left(\left(N_{1}, \cdots, N_{k-1}\right)=\left(n_{1}, \cdots, n_{k-1}\right) \mid\left(I_{1}, \cdots, I_{k}\right)=\left(i_{1}, \cdots, i_{k-1}\right)\right)>0
$$

within statistical distance $(2 \beta)$ from $\left\{P_{i_{k}}^{n}\right\}_{n}$, the conditional distribution is

$$
\left\{P\left(N_{1}, \cdots, N_{k-1}\right)=\left(n_{1}, \cdots, n_{k}\right) \wedge N_{k}=n \mid\left(I_{1}, \cdots, I_{k}\right)=\left(i_{1}, \cdots, i_{k}\right)\right\} n
$$

Please note that an easy argument shows that a device with noise level a must achieve an expected score of at least wg- 1 .

Now comes to discussing completeness.

Suppose that $S_{1}, S_{2}, \cdots, S_{Y}$ is $B$ Martingale in entropy evolution with $\left|S_{i+1}-S_{i}\right| \leq 1$, and $\operatorname{Var}\left[S_{i+1}-S_{i} \mid S_{i}, \cdots, S_{i}\right] \leq w$, for all $i, 1 \leq i \leq N-1$. Then, for any $\lambda \in(0,1)$, there is

$$
P\left[S_{Y} \geq \lambda W Y\right] \leq \exp \left(-\lambda^{2} \frac{w}{2} Y\left(1-\frac{1-w}{3} \lambda\right)\right)
$$

Particularly, if $\lambda \leq 1$, we have that

$$
P\left[S_{Y} \geq \lambda W Y\right] \leq \exp \left(-\lambda^{2} \frac{w}{2} Y\right)
$$

Let the quantum device in quantum information system has noise level $\mu^{\prime}<\mu$. So, the probability of an abort is at most $\exp \left(-\left(\mu-\mu^{\prime}\right)^{2} q Y / 3\right)$.

Suppose that $I_{1}, \cdots, I_{Y}$ and $N_{1}, \cdots, N_{Y}$ as random variables which contain the inputs and outputs for system $R$. Suppose that $Z_{i}$ is equal to 1 if won on the ith round in the game, and 0 otherwise. Let

$$
Z_{i}=E\left[Z_{i} \mid I_{1}, \cdots, I_{i}-1, N_{1}, \cdots, N_{i}-1\right]
$$

By definition, system $R$ is aborted when [11]

$$
\sum_{i} g_{i}\left(1-z_{i}\right) \geq\left(1-W_{G}+\mu\right) q Y
$$


Through assumption,

$$
\sum_{i}\left(W_{G}-z_{i}\right) \mu^{\prime} \leq y
$$

Suppose

$$
R_{i}=\sum_{k=1}^{i} g_{k}\left(1-Z_{k}\right)-q \sum_{k=1}^{i}\left(1-Z_{k}\right)
$$

Then, $R_{1}, R_{2}, \cdots$ is a Martingale with

$$
\operatorname{Var}\left[R_{i}-R_{i-1} \mid R_{1} \cdots R_{i-1}\right]=q\left(1-z_{i}\right)\left[1-q\left(1-Z_{i}\right)\right] \leq q
$$

thus, Equation (42) can be expressed as

$$
\sum_{i} g_{i}\left(1-Z_{i}\right)-q \sum_{i}\left(1-Z_{i}\right) \geq \mu q Y-q \sum_{i}\left(W_{G}-Z_{i}\right) \geq\left(\mu-\mu^{\prime}\right) q Y
$$

Therefore, the stability probability of quantum entropy is $\leq \exp \left(-\left(\mu-\mu^{\prime}\right)^{2} q Y / 3\right)$.

\section{Conclusion}

In this work, the quantum entropy is employed to evaluate the uncertainty of quantum information system. First, a quantum information system under uncertain environment is modelled as quantum entropy system. Second, entropy measurement of quantum information system is different from classical model. Third, for improvement for quantum information system, the entropy evolution strategy of quantum information system is provided. More importantly, different form additional features in previous works, quantum entropy can provide us a true random to describe the uncertainty by quantum mechanics.

\section{Acknowledgements}

This research was supported by the National Natural Science Foundation of China (No. 71471102), and Yichang University Applied Basic Research Project in China (Grant No. A17-302-a13).

\section{References}

[1] Li, J.J., Zhang, R.B. and Yang, Y. (2017) Multi-AUV Autonomous Task Planning Based on the Scroll Time Domain Quantum Bee Colony Optimization Algorithm in Uncertain Environment. PLOS One, 12, e0188291. https://doi.org/10.1371/journal.pone.0188291

[2] Lyons, D.M., Arkin, R.C. and Jiang, S. (2017) Performance Verification for Robot Missions in Uncertain Environments. Robotics and Autonomous Systems, 98, 89-104. https://doi.org/10.1016/j.robot.2017.07.001

[3] Li, Y.-H., Li, X.-L. and Wang, Z.-S. (2017) Quantum Information Splitting of an Arbitrary Three-Ion State in Ion-Trap System. International Journal of Theoretical Physics, 54, 3850-3859. https://doi.org/10.1007/s10773-015-2626-y

[4] Wang, G.-Y., Guo, Y.-N. and Zeng, K. (2015) Dynamics of Quantum Fisher Information in a Two-Level System Coupled to Multiple Bosonic Reservoirs. Chinese Physics B, 24, 1674-1056. https://doi.org/10.1088/1674-1056/24/11/114201

[5] Zhang, F.-Y., Chen, X.-Y. and Li, C. (2015) Long-Distance Quantum Information 
Transfer with Strong Coupling Hybrid Solid System. Scientific Reports, 5, 17025. https://doi.org/10.1038/srep17025

[6] Frenkel, P.E. and Weiner, M. (2015) Classical Information Storage in an n-Level Quantum System. Communications in Mathematical Physics, 340, 563-574. https://doi.org/10.1007/s00220-015-2463-0

[7] Li, C.-M., Chen, Y.-N. and Lambert, N. (2015) Certifying Single-System Steering for Quantum-Information Processing. Physical Review A, 92, Article ID: 062310. https://doi.org/10.1103/PhysRevA.92.062310

[8] Portmann, C., Matt, C. and Maurer, U. (2017) Causal Boxes: Quantum Information-Processing Systems Closed Under Composition. IEEE Transactions on Information Theory, 63, 3277-3305. https://doi.org/10.1109/TIT.2017.2676805

[9] Anwar, S.J., Ramzan, M. and Khan, M.K. (2017) Dynamics of Entanglement and Quantum Fisher Information for N-Level Atomic System under Intrinsic Decoherence. Quantum Information Processing, 16, UNSP 142.

[10] Luitz, D.J. and Bar Lev, Y. (2017) Information Propagation in Isolated Quantum Systems. Physical Review B, 96, Article ID: 020406.

https://doi.org/10.1103/PhysRevB.96.020406

[11] Nemoto, K., Devitt, S. and Munro, W.J. (2017) Noise Management to Achieve Superiority in Quantum Information Systems. Philosophical Transactions of the Royal Society A-Mathematical Physical and Engineering Sciences, 375, Article ID: 20160236. https://doi.org/10.1098/rsta.2016.0236

[12] Banuls, M.C., Yao, N.Y. and Choi, S. (2017) Dynamics of Quantum Information in Many-Body Localized Systems. Physical Review B, 96, Article ID: 174201. https://doi.org/10.1103/PhysRevB.96.174201

[13] Arefeva, I.Ya., Volovich, I.V. and Inozemcev, O.V. (2017) Holographic Control of Information and Dynamical Topology Change for Composite Open Quantum Systems. Theoretical and Mathematical Physics, 193, 1834-1843. https://doi.org/10.1134/S0040577917120091

[14] Chen, H.B., Chen, G.Y. and Chen, Y.N. (2017) Thermodynamic Description of Non-Markovian Information Flux of Nonequilibrium Open Quantum Systems. Physical Review A, 96, Article ID: 062114. https://doi.org/10.1103/PhysRevA.96.062114

[15] de la Rosa, G., Jose, J., Perez, A., Agustin, Salas, P. and Carlos, J. (2015) A Novel Measurement Method for Transient Detection Based in Wavelets Entropy and the Spectral Kurtosis: An Application to Vibrations and Acoustic Emission Signals from Termite Activity. Measurement, 68, 58-69. https://doi.org/10.1016/j.measurement.2015.02.044

[16] Uspenskaya, I.A. and Kulikov, L.A. (2015) Method for the Estimation of Standard Entropy of Crystal Phases at 298.15 K on the Limited Temperature Range of Heat Capacity Measurements. Journal of Chemical \& Engineering Data, 60, 2320-2328. https://doi.org/10.1021/acs.jced.5b00217

[17] Zhang, S., Liu, W. and He, Q. (2017) Constructing a Measurement Method of Differences in Group Preferences Based on Relative Entropy. Entropy, 19, 24. https://doi.org/10.3390/e19010024

[18] Zhang, Z., Jiang, C. and Zhang, Y. (2017) Virtual Entropy Generation (VEG) Method in Experiment Reliability Control: Implications for Heat Exchanger Measurement. Applied Thermal Engineering, 110, 1476-1482. https://doi.org/10.1016/j.applthermaleng.2016.09.051

[19] Salmani, M.H., Eshghi, K. and Neghabi, H. (2015) A Bi-Objective MIP Model for 
Facility Layout Problem in Uncertain Environment. International Journal of Advanced Manufacturing Technology, 81, 1563-1575.

https://doi.org/10.1007/s00170-015-7290-0

[20] Shen, Y. and Yao, K. (2016) A Mean-Reverting Currency Model in an Uncertain Environment. Soft Computing, 20, 4131-4138.

https://doi.org/10.1007/s00500-015-1748-8

[21] Castillo Atoche, A., Carrasco Alvarez, R. and Palma Marrufo, O. (2017) An Efficient Systolic Array Grid-Based Structure of the Robust Bayesian Regularization Technique for Realtime Enhanced Imaging in Uncertain Remote Sensing Environment. Journal of Real- Time Image Processing, 13, 783-796. https://doi.org/10.1007/s11554-014-0441-y

[22] Lefebvre, D. (2017) Dynamical Scheduling and Robust Control in Uncertain Environments with Petri Nets for DESs. Processes, 5, 54.

[23] Wang, G.-Y., Yuan, J.-B. and Zeng, H.-S. (2015) Discontinuity and Protection of Quantum Fisher Information for a Two-Qubit System. Communications in Theoretical Physics, 64, 495-500. https://doi.org/10.1088/0253-6102/64/5/495 